Revue scientifique sur la conception et l'aménagement de l'espace

\title{
Comment un diagnostic territorial partagé à partir du paysage peut-il contribuer à développer une intelligence commune du territoire?
}

How can a shared territorial diagnosis based on the landscape contribute to developing a common intelligence of a territory?

\section{Christine Partoune}

\section{(2) OpenEdition}

\section{Journals}

Édition électronique

URL : http://journals.openedition.org/paysage/1071

DOI : 10.4000/paysage. 1071

ISSN : 1969-6124

Éditeur :

École nationale supérieure du paysage de Versailles-Marseille, Institut national des sciences appliquées Centre Val de Loire - École de la nature et du paysage, École nationale supérieure d'architecture et de paysage de Bordeaux, École nationale supérieure d'architecture et de paysage de Lille, Agrocampus Angers

\section{Référence électronique}

Christine Partoune, «Comment un diagnostic territorial partagé à partir du paysage peut-il contribuer à développer une intelligence commune du territoire ? ", Projets de paysage [En ligne], 18 | 2018, mis en ligne le 11 juillet 2018, consulté le 28 novembre 2019. URL : http://journals.openedition.org/paysage/ 1071 ; DOI : 10.4000/paysage.1071

Ce document a été généré automatiquement le 28 novembre 2019.

Projets de paysage 


\section{Comment un diagnostic territorial partagé à partir du paysage peut-il contribuer à développer une intelligence commune du territoire?}

How can a shared territorial diagnosis based on the landscape contribute to developing a common intelligence of a territory?

\section{Christine Partoune}

Depuis que la participation citoyenne s'est institutionnalisée au début du millénaire sous l'impulsion du Programme des Nations unies pour le développement (PNUD) ${ }^{1}$, du Programme des Nations unies pour l'environnement (PNUE) ${ }^{2}$ et du livre blanc sur la gouvernance locale de l'Union européenne ${ }^{3}$, qui en appellent à instaurer des systèmes de gouvernance plus démocratiques, les processus d'élaboration des programmes de développement local doivent intégrer la participation de toutes les parties prenantes (Stocker, 2000).

2 Certaines institutions territoriales sont restées attachées à un " modèle hiérarchique " classique (Calon, 1997) et se conforment aux obligations officielles minimales : informer la population et recueillir son avis. Mais peu à peu ont émergé des « modèles négociés » de participation (ibid.), qui prévoient d'impliquer les citoyens dans la réalisation d'un état des lieux (on parle alors de «diagnostic territorial partagé »), voire dans la production d'un projet (Declève et al., 2002) et dans la gestion de sa mise en œuvre. Effectivement, des «diagnostics marchants» (Cordier et Géronnez, 2005), des « marches exploratoires » (Clette et al., 2007) ou des méthodes plus originales comme la "cartographie sensible» du territoire (Olmedo, 2017) se sont multipliés, ont été réalisés de manière professionnelle ou de façon empirique par des associations, des 
structures mandatées par l'État, des bureaux d'étude, des services communaux, ou encore des étudiants.

Cette ambition démocratique portée par le secteur du développement territorial est venue en quelque sorte percuter le travail des acteurs du secteur de l'éducation permanente (équivalent, en Belgique, de l'éducation populaire en France). Ce secteur a en effet pour mission historique de développer les capacités de citoyenneté active des adultes via une prise de conscience et une connaissance critique des réalités de la société, ainsi que par le développement de capacités d'analyse, de choix, d'action et d'évaluation ${ }^{4}$. Aujourd'hui, la vague de la gouvernance territoriale a gagné ce secteur et, depuis 2013, en Fédération Wallonie-Bruxelles, c'est aux centres culturels, aux maisons de la culture et aux bibliothèques qu'il est désormais demandé de réaliser une " analyse partagée du territoire ${ }^{5}$ afin de faire émerger les enjeux de société sur la base desquels sera construit leur programme pluriannuel.

La multiplication des structures qui doivent réaliser un diagnostic territorial partagé a engendré une demande de formation en la matière, ainsi que des recherches en didactique, le plus souvent sous la forme de recherches-actions.

Les missions de l'Institut d'Éco-Pédagogie- association sans but lucratif spécialisée en éducation relative à l'environnement reconnue en éducation permanente - s'inscrivent dans cette mouvance. Des modules de formation dédiés à l'approche du paysage y sont organisés depuis 2001 dont une recherche-action en didactique du paysage, intitulée Hyperpaysage ${ }^{6}$, menée en partenariat avec le Laboratoire de méthodologie de la géographie de l'université de Liège : elle porte sur la création de visites virtuelles de paysage par les apprenants pour les motiver à s'intéresser et à s'impliquer dans leur environnement tout en développant chez eux une pensée systémique et complexe (Partoune, 2004 ; Partoune et Ericx, 2005). Initiée pour l'enseignement secondaire, puis élargie à la formation initiale des enseignants du primaire, la recherche a également exploré le potentiel de l'outil hyperpaysage comme ferment de cohésion sociale et comme méthode de diagnostic territorial partagé (Partoune et Ericx, 2009d).

De 2007 à 2009, l'IEP a également participé, avec l'Unité de géographie économique et sociale de l'université de Liège, l'Instituut voor Sociale en Economische Geografie de la Katholieke Universiteit Leuven et l'association Voorming-Plus Antwerpen, à une recherche-action intitulée Topozym ${ }^{7}$, portant sur la participation citoyenne dans la gestion et l'aménagement des espaces publics dans une perspective de développement soutenable. Cette recherche visait la production d'un référentiel de formation pour des animateurs du territoire chargés de concevoir et de mettre en œuvre des processus de participation citoyenne au niveau local. Conçu comme un tableau de bord dénommé " Participation et espaces publics ${ }^{8}$ ", ce référentiel propose une réflexion de fond sur la participation citoyenne, des fiches synthétiques sur une série de concepts clés (dont le concept d'intelligence commune du territoire, qui sera développé infra), des fiches outils et la présentation des études de cas réalisées, avec la description des différents outils de diagnostic partagé qui ont été testés.

7 Depuis 2015, l'IEP s'appuie sur les résultats de ces recherches pour proposer des modules de formation à la mise en œuvre d'un diagnostic territorial partagé au départ du paysage. Le fil conducteur de ces modules est articulé autour de la question suivante: "Comment le paysage peut-il devenir le ferment d'une participation citoyenne intelligente?». 
8 Notre propos, à travers cet article, est de partager l'analyse réflexive portant sur ce dispositif de formation. Nous commencerons par définir le cadre de références qui permet de situer les paradigmes pédagogiques sur lesquels les formateurs de l'IEP s'appuient. Nous décrirons ensuite le dispositif de formation au diagnostic partagé pour illustrer comment les formateurs ont concrétisé leur recherche de cohérence entre les finalités de la formation, les objectifs à atteindre et la méthodologie mise en place. Enfin, nous proposerons une analyse des points forts et des limites de la démarche, ainsi que des pistes d'amélioration qui en émergent.

\section{Cadre de références}

9 L'analyse de tout dispositif de formation implique d'abord de clarifier le cadre paradigmatique, auquel se réfèrent les formateurs qui l'ont conçu, qui sous-tend la définition des finalités d'une formation. En l'occurrence, nous poserons trois références de cadrage :

- les fondements de l'éducation relative à l'environnement ;

- les enjeux d'une éducation relative au paysage ;

- l'intelligence commune du territoire.

\section{Les fondements de l'éducation relative à l'environnement (ErE)}

L'expression éducation relative à l'environnement est issue du monde francophone, tandis que le monde anglo-saxon s'en tient à l'expression environmental education.

11 Depuis sa création en 1991, l'Institut d'Éco-Pédagogie a profilé ses missions en accord avec les grands principes de l'éducation relative à l'environnement (parmi d'autres : Sauvé, 1994/1997 ; Villemagne, 2010), définis à plusieurs reprises au plan international, sous l'égide de l'ONU, notamment à Tbilissi en 1977.

12 L'objectif fondamental de l'ErE est « d'amener les individus et les collectivités à saisir la complexité de l'environnement tant naturel que créé par l'homme, complexité qui tient à l'interaction de ses aspects biologiques, physiques, sociaux, économiques et culturels ; ainsi qu'à acquérir les connaissances, les valeurs, les comportements et les compétences pratiques nécessaires pour participer de manière responsable et efficace à la prévention et à la solution des problèmes de l'environnement et à la gestion de la qualité de l'environnement » (Unesco-PNUE, 1977).

On le voit, l'axe " participation citoyenne » était déjà bien présent, et la filiation de la Convention européenne du paysage avec les grands défenseurs de l'environnement de l'époque est évidente. L'IEP se rallie à la vision de l'ErE défendue par Lucie Sauvé, qui estime que l'objet de l'ErE concerne le réseau de relations entre les personnes, leur groupe social d'appartenance et l'environnement, ce dernier étant vu comme le milieu de vie partagé ; en cela, il ne s'agit pas d'une forme "d'éducation à... ", mais d'une dimension essentielle de l'éducation fondamentale (Sauvé, 2002). Car, en effet, la relation que chaque individu noue avec son milieu de vie participe à la construction de son identité territoriale et à son sentiment d'appartenance à la biosphère, conditions pour que se développe une responsabilité envers les êtres vivants autres qu'humains. 
Les partisans de cette vision de l'ErE s'efforcent de combiner deux perspectives :

- une éducation pour l'environnement, centrée sur une meilleure prise en compte de l'environnement par un individu ou un groupe social afin d'en préserver ou d'en développer les qualités ;

- une éducation par l'environnement, centrée sur la personne ou le groupe social, qui reconnaît l'environnement non seulement comme un terrain particulièrement motivant pour le développement personnel, mais surtout comme un cadre de vie existentiel, un territoire de socialisation et un territoire d'éducation à la citoyenneté.

L'ErE est donc étroitement liée aux missions fondamentales de l'éducation permanente, puisqu'elle vise à induire des dynamiques sociales, d'abord à l'échelle des communautés puis à celle de réseaux de solidarité élargis, favorisant l'approche collaborative et critique des réalités socioenvironnementales et une prise en charge autonome et créative des problèmes qui se posent et des projets qui émergent (Sauvé, 2002; Orellana, 2005).

16 Sur le plan méthodologique, les principes suivants, résumés par Lucie Sauvé (1994/1997), constituent les fondements de l'ErE pratiquée à l'IEP :

- partir de l'expérience concrète ;

- privilégier une pédagogie de terrain ;

- adopter une approche interdisciplinaire et systémique ;

- favoriser l'implication active des apprenants à la construction du savoir ;

- stimuler le travail coopératif ;

- privilégier une orientation communautaire pour résoudre des problèmes environnementaux communautaires.

\section{Les enjeux d'une éducation relative au paysage}

L'éducation relative au paysage constitue l'une des dimensions essentielles de l'ErE, qui transparaît dans le préambule de la Convention européenne du paysage, où l'on peut lire que pour les États membres du Conseil de l'Europe qui l'ont signée, le paysage «représente une composante fondamentale du patrimoine naturel et culturel de l'Europe, contribuant à l'épanouissement des êtres humains [...], reconnaissant que le paysage est partout un élément important de la qualité de vie des populations, [...] persuadés que le paysage représente un élément essentiel du bien-être individuel et social » (Conseil de l'Europe, 2000, p. 1).

La vision du paysage comme " cadre de vie » et comme "miroir de la société qui y vit » constitue un socle de référence pour l'IEP, avec trois dimensions corollaires sur le plan méthodologique :

- l'approche sensible du paysage (Lassus, 1991), qui va bien au-delà d'une simple approche sensorielle (Bouillet, 1999) : fondée sur la dimension émotionnelle et affective des liens qui nous unissent en tout état de cause à nos différents cadres de vie (Loiseau et al., 1993 ; Claval, 2000), cette dimension est profondément humaniste et existentielle car elle touche à notre histoire et à notre géographicité (Dardel, 1952 ; Raffestin, 1989), à notre identité territoriale profonde (entre autres : Lipietz, 1994 ; Lüginbuhl, 1998 ; Besse, 2000 ; Lazzarotti, 2002) ;

- l'approche cognitive du paysage, plus classique, fondée sur la capacité à lire et à interpréter la physionomie des lieux et la dynamique du territoire qu'ils révèlent ;

- l'approche citoyenne (Damien, 1999), qui interroge les choix posés, les valeurs dominantes et les rapports de force mis en lumière par les paysages (Claval, 2000). Cette dimension est 
clairement politique et critique et les questions posées sont fortes (Bédard, 2009 ; Sgard, 2010) : à qui appartient le paysage ? Fait-il partie des biens communs ou autorise-t-on certains à se l'approprier, quitte à priver les autres de sa jouissance? Qui peut décider de son devenir ?, etc. formation analysé, nous verrons qu'elles sont interconnectées. En effet, la recherche Topozym a permis de constater à quel point nos espaces publics au sens strict, nos « biens communs » ordinaires, dont le paysage fait partie, ont besoin, pour être adoptés par les citoyens, que naisse chez ces derniers un sentiment d'attachement suffisamment fort. Certes, les paysages qui nous entourent nous marquent affectivement, mais nous n'en avons qu'une conscience fugace et ils composent largement, à notre insu, un univers de représentations du monde culturellement déterminé, où se forgent nos opinions, nos préjugés, nos valeurs, et nos décisions (Loiseau et al., 1993 ; Claval, 2000, 2012), que nous intégrons implicitement comme un référentiel normatif. D'où l'importance d'accorder toute notre attention à cette dimension subjective de la relation au paysage, qui reste souvent masquée dans les débats où la rationalité des arguments prévaut (Loiseau et al., 1993). La prise en compte du rapport implicite à la norme amène aussi à intégrer, dans les dispositifs de diagnostic paysager, un axe émancipatoire jugé essentiel. Sans cela, la participation citoyenne risque fort de conduire à une consolidation des idées les plus conservatrices en matière de paysage, à une banalisation de ce qui est considéré comme ayant de la valeur, ou encore à la destruction de joyaux ou de traces du passé qu'il est improbable d'apprécier sans éducation culturelle.

Enfin, la dimension cognitive de l'éducation au paysage suppose de ne pas négliger la dimension affective qui devrait y être associée, pour que la compréhension du monde dans lequel nous vivons s'accompagne d'émotions potentiellement puissantes lorsque l'on prend conscience, à sa juste mesure, de l'immense labeur des hommes qui ont sculpté le substrat originel, du caractère précieux de la couche arable, si fine et si fragile, tout comme de l'impressionnante histoire des roches et des formes du relief.

\section{L'intelligence commune du territoire}

Les chercheurs Topozym se sont posé la question suivante : «Comment éviter que les diagnostics réalisés ne servent qu'à renforcer le pouvoir de celles et de ceux qui le détiennent déjà par ailleurs, en d'autres lieux ? » Une réponse à cette question a abouti à la définition des attributs du concept d' "intelligence commune du territoire" (Partoune, 2009b et 2011-2012) en s'appuyant sur le cadre théorique posé par le pédagogue américain Howard Gardner à propos des multiples formes de l'intelligence. L'intelligence est à prendre au sens de "capacité à résoudre des problèmes ou à produire des biens ayant une valeur dans un contexte culturel ou collectif précis ${ }^{9}$.» (Gardner, 1983/2003, p. 81) L'intelligence territoriale a été appréhendée sous l'angle des compétences collectives d'un groupe d'acteurs, c'est pourquoi nous parlons d'intelligence commune du territoire, qui pourrait être définie comme la "capacité d'un groupe d'acteurs à résoudre des problèmes qui se posent sur un territoire donné ou à y développer des projets, d'une manière qui soit reconnue comme valable par les personnes concernées » (Partoune, 2009b, p. 1).

Projets de paysage, $18 \mid 2018$ 
La recherche a abouti à la formulation d'une liste de critères (toujours ouverte) qui permettrait d'identifier la façon de reconnaître un groupe d'acteurs qui aurait développé une intelligence commune du territoire.

Critères pour reconnaître qu'un groupe d'acteurs a développé une intelligence commune de son territoire

Un soutien politique garanti (existence d'instances de concertation, suivi des projets par des élus et des fonctionnaires, soutien financier...).

Le plaisir, la reconnaissance mutuelle (Scheers, 2009b), la connivence entre toutes les parties prenantes.

Une connaissance mutualisée des lieux et des gens (Lardon, 2006), voire un ancrage territorial commun.

L'existence perceptible d'un référentiel-qualité du territoire partagé et promu par un grand nombre d'acteurs différents (Brédif, 2008).

Le désir et la capacité d'ouverture et de dialogue entre personnes ayant des points de vue différents, voire opposés, conduisant à constituer un réseau et une communauté territoriale solidaire.

Une éthique commune (Hansotte, 2005), se traduisant notamment par le souci d'intégrer la participation des « gens silencieux » (Scheers, 2009a), des « sansvoix », de manière directe ou indirecte.

Le souci de la présence des «non-humains » sur le territoire, le souci de préserver la nature et l'environnement (Cottereau, 2005).

Une distance critique partagée (Brédif, 2004) et une approche systémique du territoire (Rosnay, 1975 ; Michelin et Joliveau, 2005 ; Moine, 2006 ; Partoune, 2009a), impliquant la capacité de chacun de se comporter comme micro et comme macro-acteur (Ollagnon, 1987).

L'existence d'un document qui traduise une vision commune et durable du territoire ; une mobilisation à long terme pour la mettre en œuvre (Lardon et Piveteau, 2005).

Le souci d'assurer la transmission de l'intelligence commune du territoire développée.

Ces critères peuvent servir de balises pour concevoir un processus d'accompagnement des parties prenantes d'un territoire. Certains charpentent le dispositif de formation décrit et analysé plus loin, c'est pourquoi ils sont davantage explicités ci-dessous.

- Un ancrage territorial commun et un référentiel qualité conscientisé collectivement

Une intelligence commune du territoire se développe d'autant mieux lorsque les acteurs concernés partagent un même attachement pour le territoire, surtout lorsque les acteurs ont construit ensemble cet ancrage affectif et leurs connaissances à propos du territoire, et qu'ils disposent d'un vocabulaire commun pour parler des lieux et des gens. Ainsi émerge alors un référentiel qualité commun sur lequel se greffe une identité 
collective : les habitants sont conscients des atouts de leur territoire, ils sont fiers d'en parler et désireux d'en préserver ou d'en améliorer les qualités.

- Le plaisir, la reconnaissance mutuelle, la connivence entre tous

Pour qu'advienne une intelligence commune du territoire qui soit au service de tous au sein d'un groupe d'acteurs, il convient que ces derniers soient unis sur le plan affectif et aient du plaisir à se retrouver. Mais pour être capables d'œuvrer ensemble, cela doit aller plus loin: il convient que les parties prenantes se reconnaissent mutuellement comme « interlocuteurs valables ».

- Le souci des non-humains

Un groupe d'acteurs qui a atteint un haut niveau d'intelligence commune du territoire se reconnaît au fait de manifester un respect et une compréhension des préoccupations et des revendications de ceux, parmi ses membres, qui défendent «la nature ». Ils reconnaissent comme fondamentale la relation profonde qui les lie à leur environnement naturel.

- Une éthique commune et le souci d'intégrer la participation des « gens silencieux "

Le groupe est capable d'autoréflexivité et questionne sa légitimité à définir un projet de territoire. Il cherche à élucider ses cadres de référence implicites et est ouvert à la remise en question. Il sait aller au-delà des intérêts particuliers pour trouver des voies originales servant aussi l'intérêt général (Hansotte, 2005). En accord avec cet idéal, le groupe manifeste un réel souci d'accueil et d'intégration des gens habituellement «silencieux » qui se trouvent en marge du processus. À cette fin, il accepte que des méthodes spécifiques soient mises en œuvre pour les aider à participer et à assurer que ce qui s'exprime puisse être entendu et pris en compte par ceux qui ont le pouvoir de décision (Scheers, 2009a, p. 2).

\section{Le diagnostic partagé, une opportunité pour développer une intelligence commune du territoire}

La formation au diagnostic territorial partagé proposée par l'IEP précise d'emblée que la finalité de ce dernier est de construire une intelligence commune du territoire fondée sur les valeurs de l'éducation permanente, tout autant, sinon bien plus, que de permettre d'identifier les problèmes qui se posent sur un territoire et les menaces qui pèsent sur son devenir.

Le public qui s'inscrit à cette formation est principalement composé de personnes qui ont une fonction d'animateur du territoire en milieu rural ou urbain, ou de personnes qui doivent réaliser un diagnostic territorial partagé pour construire leur plan d'action (centres culturels...).

La méthodologie globale du module de 4 jours est fondée sur une construction progressive des références à acquérir par le biais d'activités diverses mettant les participants à contribution et encourageant l'apprentissage coopératif (Abrami et al., 1996): recueil et analyse de représentations (Sauvé et Machabée, 2000), expérimentations d'outils et de méthodes d'animation suivies d'une analyse réflexive, temps de structuration. 
L'analyse de la chronologie des étapes du processus de formation fait apparaître un modèle d'intervention pédagogique original passant par trois étapes clés (figure 1) :

- une phase d'immersion visant la mise en relation des personnes entre elles, avec les autres et avec le milieu de vie ;

- une phase d'éveil et de prise de conscience de soi au monde reposant sur une approche globale de la personne dans sa relation au milieu de vie ;

- une phase de développement de l'intelligence citoyenne intégrant une approche systémique et complexe du milieu de vie, ainsi que l'émancipation socioculturelle des personnes et des groupes sociaux qui participent au diagnostic.

Figure 1. Fil conducteur du module de formation au diagnostic territorial partagé proposé par l'IEP

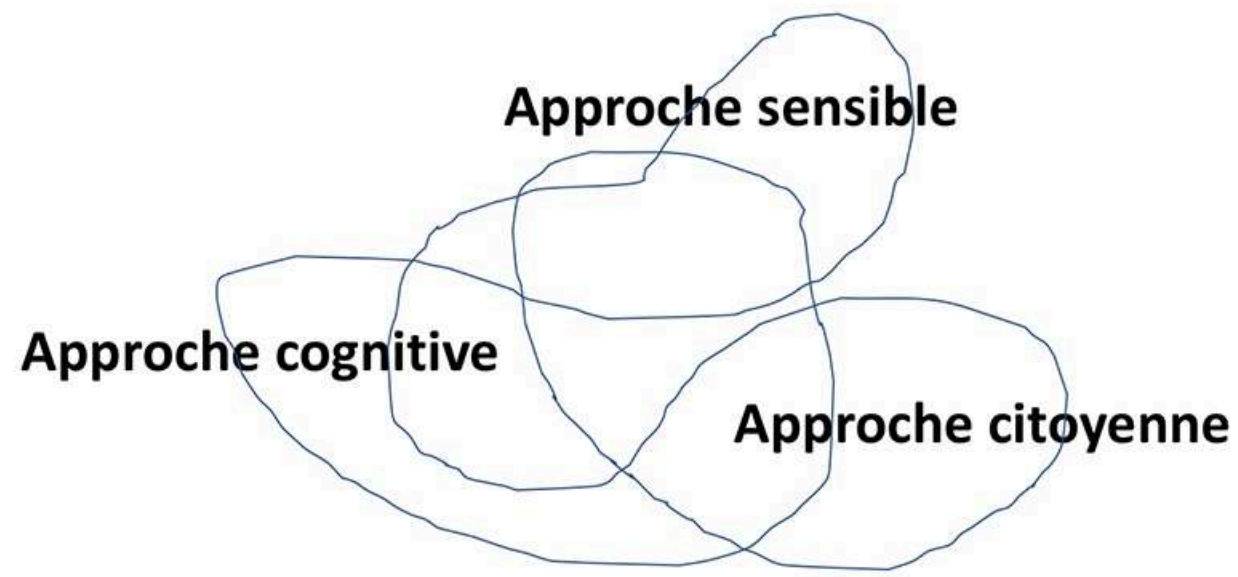

\section{Immersion}

La phase d'immersion a pour but d'activer les liens de chacun avec le sujet abordé et de favoriser la rencontre avec les autres, dans une ambiance ludique instaurant d'emblée un climat convivial.

La formation démarre par une présentation qui se limite à dire d'où chacun vient et à pointer cet endroit sur une carte. Au fur et à mesure, les autres participants sont invités à exprimer par mots-clés ce que ce lieu évoque pour eux, dans trois registres (perception - opinion - attachement), après quoi la personne concernée livre ce qu'il en est pour elle. Ce faisant, une moisson de fragments de représentations paysagères mentales est récoltée. L'analyse réflexive amène à poser au groupe deux questions : qui a le droit de réaliser un diagnostic sur un territoire donné ? Les acteurs externes ont-ils autant de légitimité pour le faire que les acteurs internes? Avis et témoignages sont entendus et structurés.

L'activité suivante propose à chacun de représenter par le dessin, sous forme d'une fresque chronologique, ses territoires d'appartenance. Les commentaires font apparaître les points d'ancrage de chacun, constitués d'abord par les liens sociaux noués, ensuite viennent les paysages et la qualité de l'environnement en général. L'analyse réflexive met en lumière à la fois l'importance de ces derniers éléments, tout autant que la difficulté de chacun à évoquer précisément ce qui fonde l'attachement au paysage et à 
l'environnement : la relation de chacun à l'environnement apparaît comme étant assez floue, et les participants expriment souvent qu'au fond ils ne se sont jamais posé ce genre de question.

La clôture de cette phase d'immersion donne du sens à la phase suivante. Si l'on part de l'hypothèse que, pour beaucoup de personnes, la relation avec le milieu de vie est certes là, mais qu'elle est enfouie dans l'inconscient, ou encore que le milieu de vie est occupé et parcouru avec indifférence, dans une espèce de non-présence de soi au monde, alors il convient de ménager une transition pédagogique avant de s'engager dans la phase de diagnostic territorial proprement dite.

\section{Éveil et conscience de soi au monde}

La phase d'éveil et de conscience de soi au monde vise clairement le développement de chaque personne dans sa relation à l'environnement, en prenant en considération le fait que nous sommes des êtres vivants. «Vivant » s'accorde avec l'idée de mouvance, de plasticité, d'émergence et de créativité. Cette vision implique de choisir de travailler sur la conscience de soi au monde dans un schéma dynamique, en proposant des outils et des méthodes à l'opposé d'un travail « en chambre " consacré à se révéler à soi par une introspection dirigée. C'est pourquoi cette phase va se dérouler à l'extérieur et c'est l'approche globale du paysage qui va servir de ferment pour commencer à bâtir concrètement une intelligence commune du territoire parcouru. Sans cette phase, un diagnostic partagé risque de conduire à quelque chose d'assez rugueux, sans profondeur, limité à quelques grandes idées toutes faites.

Une palette d'activités ludiques diversifiées et de courte durée est proposée aux participants tout au long d'un parcours dans la ville. Les approches sensorielle, sensible, imaginative, cognitive et affective sont convoquées, en variant les moyens d'expression et de communication. En voici quelques exemples.

38 La première activité va permettre d'aborder la question des filtres de la perception. Il s'agit de parcourir une portion de trajet à la façon d'un « scanner », avec une contrainte différente pour chacun, donnée à l'insu des autres : la moitié du groupe est chargée d'enregistrer en détail les caractéristiques des fenêtres, des murs et des toits (vision objective du paysage), tandis que l'autre moitié va repérer ce qui est insolite, choquant ou réjouissant (vision subjective du paysage). Lors de la mise en commun, la surprise est systématique : les participants étant concentrés sur leur mission, leur perception du paysage est devenue très sélective et les a aveuglés sur d'autres aspects. Cette prise de conscience aura un impact sur la suite du trajet: les participants vont intégrer cette manière « scanner » de parcourir les lieux.

L'activité suivante s'appuie sur le vocabulaire technique architectural pour affiner le regard et élargir la capacité à prendre du plaisir dans l'environnement. L'endroit pour mener l'activité est choisi pour la diversité des façades alentour (style, époque). L'outil utilisé est un jeu de cartes qui présente une cinquantaine de termes définis et illustrés, des plus usuels au plus rares (pilastre, linteau, judas, tabatière, chapiteau, console...). Les cartes sont réparties entre les participants qui partent à la chasse aux « mots de la maison» en observant les bâtiments. Ensuite, leur créativité est convoquée: on imagine que les façades sont douées de parole et qu'elles échangent entre elles! Dialogue amoureux, petits potins, souvenirs, histoires à dormir debout, conflits de génération... : par deux ou trois, les participants choisissent deux façades et rédigent un 
fragment de dialogue entre elles avec comme contrainte d'inclure dans le texte un maximum de termes techniques vus auparavant. L'humour est au rendez-vous, et la force de cette activité c'est véritablement de sortir les lieux de l'anonymat et de l'indifférence.

La démarche se poursuit par une approche sensorielle des couleurs du paysage. Afin d'affiner le regard et de sensibiliser les participants aux nuances de couleurs, un jeu d'une centaine de cartes colorées est mis à leur disposition afin d'en extraire les teintes qui caractérisent le paysage environnant, voire un élément remarquable de celui-ci, comme un affleurement rocheux, un vieux mur, etc. Avec cet échantillon, ils composent une œuvre abstraite (figure 2). Les émotions sont partagées. Le décodage de cette activité très plaisante laisse souvent entendre à quel point des participants qui connaissent les lieux " par cœur » les redécouvrent. Cette prise de conscience devient en elle-même une source potentielle d'approfondissement de la relation sensible à d'autres lieux, à d'autres ambiances.

Figure 2. La palette des couleurs du paysage

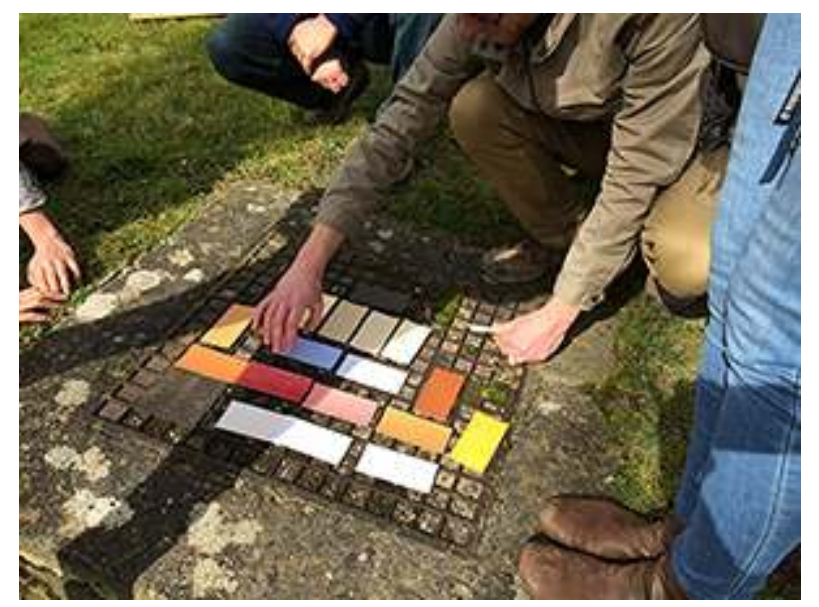

Christine Partoune, Jodoigne, 2017

Le croquis paysager a pour fonction d'élargir les capacités d'observation visuelle. Outre cet objectif, l'activité est pensée comme un "piège à désir " d'élargir le savoir. Les participants sont répartis par groupes de 4 et disposent d'une feuille A3 avec support, ainsi que de crayons de couleur et d'une gomme. Le paysage à représenter «le plus fidèlement possible " n'est pas visible de l'endroit où ils se trouvent: il faut, pour accéder au point de vue, parcourir une bonne trentaine de mètres, et chaque sousgroupe y envoie ses émissaires à tour de rôle. Le timing est imposé : chaque émissaire ne peut rester qu'une minute au point de vue; dès son retour, l'émissaire suivant peut partir vers le point de vue, tandis que celui qui est revenu donne ses instructions du mieux qu'il peut à l'un de ses coéquipiers chargé de dessiner ; ils doivent jouer chaque rôle au moins une fois. Cette activité se poursuit par une exposition des dessins réalisés et une expression du vécu, en restant à distance du paysage représenté (figure 3). Après quoi, les participants peuvent ardemment rejoindre le point de vue et prendre le temps d'observer le paysage tout à leur aise. La curiosité est très stimulée par cet exercice, et l'apprentissage de la lecture du paysage répond alors à un réel désir de découvrir l'histoire des lieux. 
Figure 3. Croquis paysagers collectifs

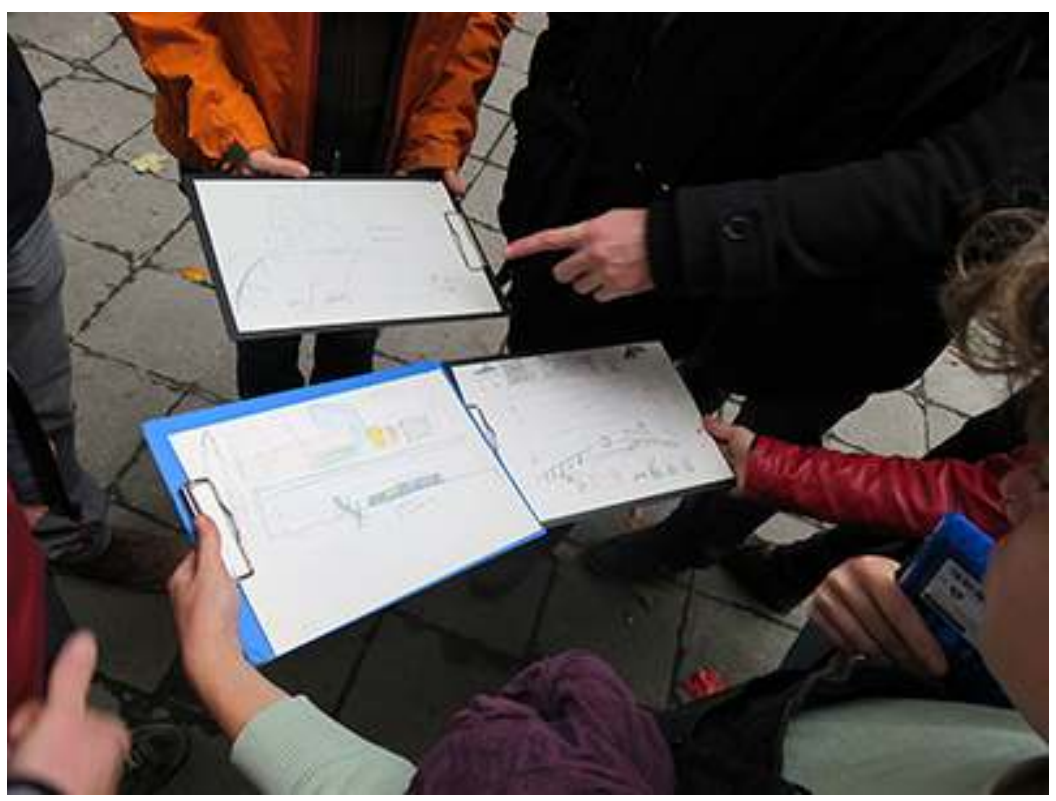

Une activité basée sur des photos anciennes vient compléter cette sensibilisation à l'histoire des lieux, par la recherche de traces du passé dans le paysage. C'est l'occasion d'acquérir des connaissances sur les grands courants urbanistiques qui ont présidé à l'aménagement des territoires, tout en prenant conscience qu'il est possible de faire bouger les choses et de faire valoir des valeurs différentes de celles qui présidaient autrefois. C'est aussi le moment d'introduire l'inéluctable débat entre anciens et modernes, par exemple au départ d'une intervention contemporaine controversée.

L'activité suivante consiste à réaliser un pastiche de paysage en se référant au courant impressionniste, avec quelques reproductions de tableaux célèbres comme sources d'inspiration, et différents outils pour dessiner (pastels, fusains, gouaches, crayons, feutres). Chacun choisit un style particulier et s'installe pour dessiner à l'aise sur un papier au format A5, plus rassurant qu'un format plus grand. L'endroit qui pourrait être considéré comme idéal pour cette activité est un agréable parc urbain d'une certaine taille offrant des recoins intimes, mais il est tout aussi intéressant de réaliser cette activité dans un lieu a priori moins charmant, pour apprendre à y poser un regard poétique (figure 4). Le partage du vécu porte sur ce qui change dans la relation au paysage et à soi-même, du fait de réaliser ce dessin, puis l'analyse réflexive est déplacée progressivement, avec quelques questions de fond: comment intégrer dans un diagnostic la capacité de sublimation des lieux par le regard artistique? Dans quelle mesure un courant artistique est-il capable d'engendrer de nouvelles visions de l'aménagement du territoire?, etc. 
Figure 4. Pastiche de paysage en bord de Meuse



La phase d'éveil se termine par une prise de selfie avec comme consigne : "Choisissez un arrière-plan paysager qui vous corresponde » (figure 5).

Figure 5. Des selfies avec un arrière-plan en accord avec sa personnalité

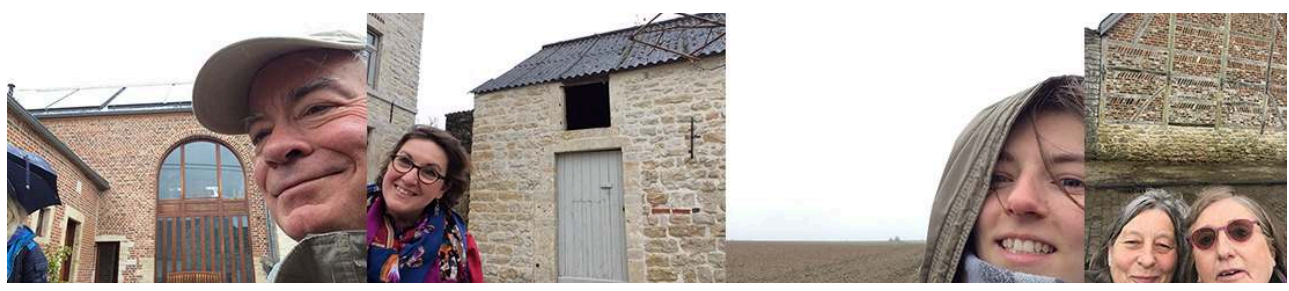

Cette phase d'éveil et de prise de conscience, riche en partages et en évocations de liens avec d'autres lieux, par effet de rebond, crée dans un groupe un climat de confiance et d'écoute. Peu à peu, naît une petite communauté d'apprentissage mutuel, avec un ancrage territorial commun. Le terreau est prêt pour accueillir la phase suivante, dédiée au développement de l'esprit critique et de l'intelligence citoyenne.

\section{Intelligence citoyenne}

La troisième phase est consacrée au diagnostic territorial en partant du paysage. Le fait de réaliser ce diagnostic sur le terrain est revendiqué comme un atout pour faciliter les échanges de points de vue et pour réduire l'écart existant entre la représentation mentale que l'on se fait d'un lieu et la perception que l'on peut en avoir sur place; cela permet aussi de mieux apprécier les enjeux et les potentialités d'un aménagement de l'espace public, tout en prenant davantage conscience de la façon dont fonctionnent les lieux à divers moments et des différences d'ambiance paysagère qui peuvent exister (Dalimier, 2009). Une bonne appréciation de la qualité du cadre de vie à l'échelle d'un quartier ou d'un village par les citoyens passe aussi par un arpentage des lieux qu'ils ne fréquentent pas spontanément dans leur vie quotidienne.

Les activités proposées s'inscrivent elles aussi dans la continuité du processus de développement d'une intelligence commune du territoire, avec de nouveaux défis: avoir le souci des « sans voix » et celui de préserver la nature ; pratiquer le changement d'échelle pour pouvoir se situer à la fois en tant que micro- et macro-acteur. 
48 différents points du territoire choisis pour leur caractère représentatif, soit le long d'un transect ou d'un parcours en boucle.

et pour accéder à une vision plus systémique du territoire. Ils sont utilisés soit en

49

\begin{abstract}
Mentionnons l'outil «jeu de cartes des enjeux du territoire» imaginé par Sylvie Lardon, qui sert de référence pour confectionner des jeux de cartes appropriés aux territoires concernés par le diagnostic (collectif IEP, 2011) ; les outils " photo aérienne " (figure 6) ou "plan de ville» (figure 7) avec gommettes de couleur par thématique qui expriment les préoccupations des citoyens (sécurité, propreté, convivialité...), et servent à pointer les lieux où les enjeux sont les plus cruciaux (ibid.); l'outil "hyperpaysage ", aussi, qui consiste à produire une visite virtuelle du territoire au départ d'images panoramiques du paysage (Partoune et Ericx, 2005), ou encore l'outil « Photolangage » (figure 8).
\end{abstract}

Figure 6. Une image aérienne pour reporter les préoccupations des citoyens

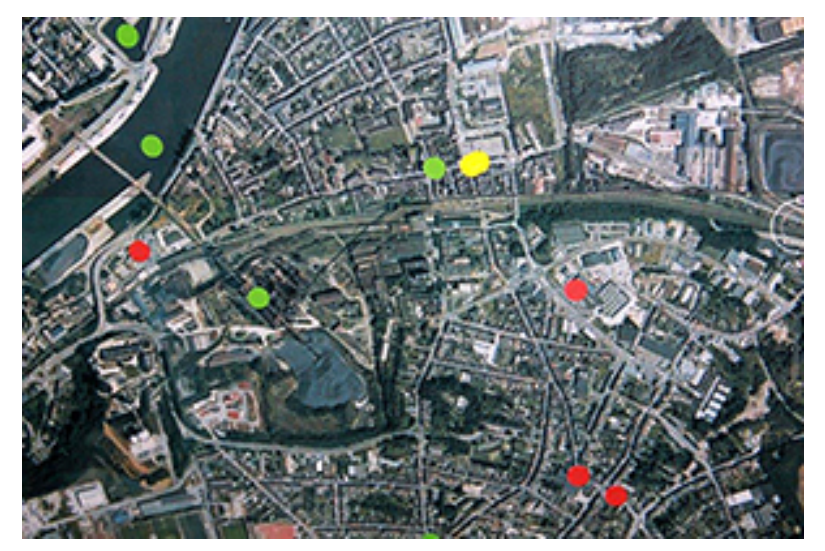

Figure 7. Report des enjeux pressentis sur un plan de ville



Projets de paysage, $18 \mid 2018$ 
Figure 8. Un photolangage pour exprimer ses préoccupations ou ses attentes

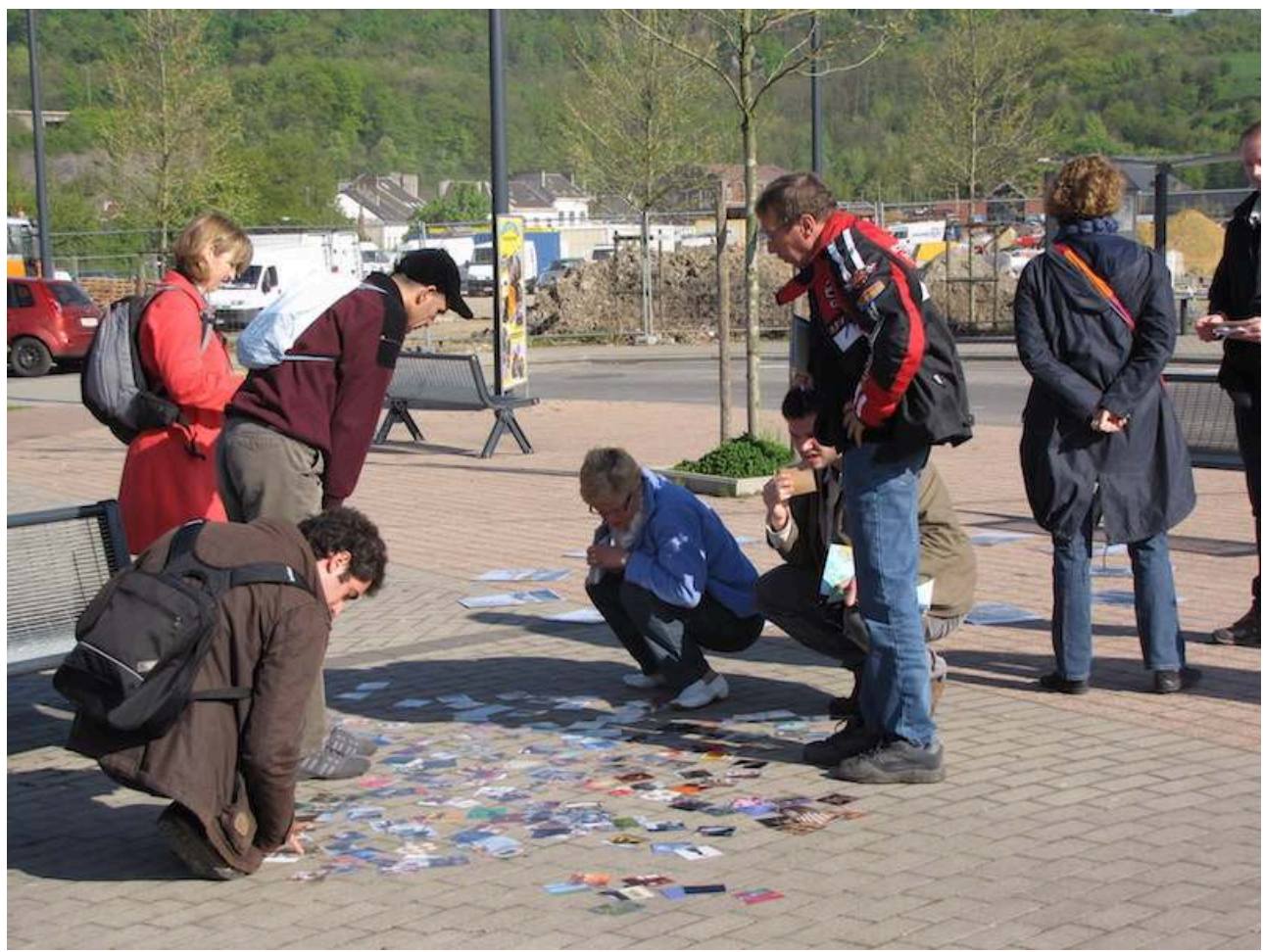

50 Afin d'introduire le souci des « sans voix », c'est le jeu de rôle qui est privilégié quand il n'est pas possible de les intégrer directement dans le processus de diagnostic territorial : chacun reçoit ou choisit un rôle (malvoyant, malentendant, personne à mobilité réduite, ado qui s'ennuie...) et va parcourir l'espace défini en essayant de se mettre à la place de la personne en question.

51 Une autre manière de travailler est d'aller à leur rencontre sur le terrain, dans les lieux qu'ils fréquentent, plutôt que d'essayer de les amener à participer à des réunions formelles qui les mettraient mal à l'aise. Des outils de médiation sont alors indispensables pour amorcer le contact et pour favoriser l'expression. Les photos de paysage sont privilégiées pour ce faire : une image panoramique imprimée au format A3 s'avère un outil intéressant dans la mesure où la vue désarçonne de prime abord (figure 9). Les participants testent le recueil d'avis sur des propositions concrètes d'aménagement alternatif suggérées par des images. À cette fin, l'outil utilisé est encore un photolangage composé d'exemples soignés d'aménagement urbain alternatif. Cette entrée en matière positive évite de tomber dans l'engrenage des discours pessimistes. L'outil vise aussi l'émancipation culturelle des citoyens et l'ouverture à la prise en considération des "sans voix " autres qu'eux-mêmes, par exemple avec des images montrant une plaine de jeu adaptée à des enfants handicapés, une pelouse sauvage, un coin lecture abrité, des toilettes publiques de qualité, des ruches, etc.

Figure 9. Une image panoramique pour faciliter le dialogue




52 C'est également par le biais du jeu de rôle que la décentration est encouragée pour accéder à un point de vue qui implique un changement d'échelle spatiale et temporelle (incarner un fonctionnaire chargé de la mobilité ou du développement touristique à l'échelle régionale, un promoteur immobilier, une structure chargée de repenser la politique du logement social, etc.).

53 Quant au souci pour la nature, il est éveillé en réalisant un petit inventaire de la biodiversité dans un lieu où elle est particulièrement préservée, puis un état des lieux $\mathrm{du}$ maillage écologique local est réalisé sur la base de photos aériennes et d'observations réalisées au départ de la rue.

54 En pratiquant sur le terrain ces changements de points de vue et de postures, l'objectif est que les citoyens puissent, d'une part, accéder à une meilleure compréhension de la complexité du système d'acteurs en présence et des enjeux qu'ils défendent et, d'autre part, acquérir la confiance dans le fait que les choses évoluent dans un sens positif. Ils devront ensuite construire une argumentation pour défendre leurs propres valeurs, mais là s'arrête le travail en prise directe avec le paysage.

\section{Forces et limites du dispositif de formation}

En quatre jours, les participants comprennent bien le sens et l'intérêt de la démarche et des outils proposés, par le fait d'avoir ressenti et pensé le territoire autrement, à partir $\mathrm{du}$ paysage, et d'avoir rapidement construit par ce biais une communauté d'apprentissages conviviale et enrichissante.

La force du dispositif repose sur plusieurs piliers :

- l'approche globale de l'apprenant, qui le sollicite à tous les niveaux de son être (approche sensible, cognitive, citoyenne) ;

- la qualité et la profondeur des interactions entre participants, grâce aux méthodes actives et participatives proposées; le caractère ludique de la plupart des activités proposées y participe aussi pleinement;

- l'attitude des formateurs (écoute, respect de chacun, capacité d'adaptation et de rebondissement) et leurs compétences pédagogiques ;

- le choix des lieux et des paysages à s'approprier pour réaliser les activités.

57 Les limites de la démarche sont liées au bagage des participants. Certains n'ont aucune formation pédagogique ni aucune expérience d'animation à leur actif. Les contenus s'avèrent dès lors très copieux et l'analyse réflexive se limite souvent à ce qui est vécu, ce qui est déjà très riche en soi, mais le transfert à des situations d'animation avec un public donné reste très limité. Ces constats débouchent souvent sur la prise de conscience qu'une formation de base en animation est nécessaire, mais à l'heure actuelle, en Belgique, elles sont réservées au secteur jeunesse. Il y a donc une réflexion à avoir sur la conception d'un curriculum de formation à l'animation territoriale qui intègre l'acquisition de compétences pédagogiques de base. Cet enjeu est d'autant plus crucial que l'on trouve sur internet un grand nombre d'outils et de méthodes qui semblent faciles à appliquer, mais les utiliser à bon escient au regard des valeurs que l'on souhaite défendre requiert une prise de recul qui ne se construit pas en un claquement de doigt.

Une autre limite importante en termes d'éthique de formation est le caractère souvent inédit, pour les participants, de l'approche systémique et complexe du territoire, de 
l'apprenant, de l'apprentissage et de l'animation territoriale. Cette approche bouscule et remet en question les croyances, les préjugés, les idées toutes faites..., à propos de soi, des autres et de l'environnement. Les ruptures vécues sont souvent fortes et le dispositif permet de les accueillir sereinement, mais le fait que la formation se déroule sur 4 jours ne permet pas de les accompagner sur le long terme. Le désir de «se revoir", de "rester en contact", qui est souvent exprimé en fin de formation, débouche rarement sur des engagements concrets. À cet égard, l'existence d'un réseau de soutien au développement professionnel des animateurs du territoire, comme il en existe pour les animateurs des Groupes d'action locale du programme LEADER de développement rural ${ }^{10}$ pourrait être considérée comme un nouvel indicateur d'une intelligence commune du territoire.

\section{Conclusion} logique d'éducation permanente, c'est une démarche qui tente de bousculer les conservatismes, les préoccupations individualistes et le pouvoir des groupes sociaux les mieux structurés et organisés. chacun noue avec son environnement, pour que celle-ci devienne plus consciente, plus compréhensive et plus profonde sur le plan de la sensibilité. Elles concernent aussi la relation que chaque personne établit avec ses "cohabitants", voisins ou usagers réguliers du territoire, à travers les activités vécues en commun dans le milieu de vie partagé. Elles exigent enfin un travail de décentration et d'émancipation socioculturelle, afin d'être en mesure de concevoir et de négocier une vision et un projet de territoire commun, bon " pour nous tous".

61 À toutes les étapes du processus d'accompagnement d'un groupe de citoyens qui s'engage dans une démarche de diagnostic, le paysage peut véritablement servir de point d'appui pour développer une intelligence commune du territoire en combinant adroitement des activités d'éducation par le paysage, au sujet du paysage et pour le paysage.

62 Cependant, utiliser le paysage "cadre de vie » comme catalyseur de reliance avec soimême, pour prendre conscience de nos représentations, de nos attaches, de nos affects et de nos valeurs, suppose de la part de l'animateur territorial d'avoir développé suffisamment son « intelligence intrapersonnelle ( Gardner, 1983/2003), avant d'être en mesure de concevoir un parcours sensible pour d'autres. Or, cette relation intime et consciente avec le paysage ne se construit pas en un jour : elle a besoin du temps du parcours curieux, du temps de la pause contemplative, du temps de l'errance réjouissante, comme du temps de l'arrêt sur image déplorable; elle a besoin d'être nourrie par des connaissances sur l'histoire du lieu et par des rencontres avec les personnes qui y vivent, pour développer des projets révélant une intelligence naturaliste-écologique approfondie du territoire. Cette expérience personnelle de l'animateur, de l'animatrice du territoire par et avec le paysage représente probablement l'un des enjeux les plus cruciaux de la formation, au regard de l'évolution culturelle ambiante où le contact direct avec le milieu de vie et le désir de le connaître s'amoindrissent fortement. 
63 Le développement de l'esprit critique et la prise en compte de la complexité des personnes et des situations représentent un autre enjeu crucial de la formation. Il n'est en effet pas facile de prendre conscience à quel point nous adoptons un schéma linéaire de pensée, une vision simpliste, peu nuancée, et sans doute fort naïve, aussi, emportés par les rêves des "demain" de tous bords. Mettre en œuvre des démarches de participation citoyenne exige de sortir d'une vision angélique de celle-ci, tout comme d'une vision idéalisée des "citoyens". Apprendre à participer en réalisant un diagnostic territorial, c'est apprendre à sortir du bois, à découvrir les résistances profondes des systèmes, à ne pas se décourager tout de suite, à se mobiliser à long terme, à collaborer, à négocier... à faire de la politique! Devenir un animateur capable d'accompagner un groupe sur le chemin politique de la définition d'un dessein commun suppose l'acquisition d'une panoplie de compétences liées au développement de l'intelligence "interpersonnelle» (Gardner, 1983/2003), compétences que le groupe devra acquérir à son tour, collectivement.

On le voit, avec les valeurs de l'éducation permanente posées comme finalités dans la réalisation d'un diagnostic partagé, croisées avec l'ambition de l'Institut d'Écopédagogie d'inscrire cette action dans une stratégie globale d'éducation relative au paysage, la mise en lumière des compétences pédagogiques et didactiques requises pour concevoir ce type de dispositifs conduit à penser qu'une formation de quatre jours suffit à peine à dresser le panorama des besoins de formation des participants. Il y a là, véritablement, de quoi composer un programme d'une année entière de spécialisation en animation territoriale.

\section{BIBLIOGRAPHIE}

Abrami, P. C., Chambers, B., Poulsen, C., Simone, C. de, Apollonia, S. d', Howden, J., L'Apprentissage coopératif. Théories, méthodes, activités, Montréal, Les Éditions de la Chenelière, 1996.

Bédard, M. (dir.), Le paysage, un projet politique, Presses de l'Université du Québec, 2009, 332 p.

Besse, J.-M., Voir la terre, six essais sur le paysage et la géographie, Paris/Versailles, Actes Sud/École nationale supérieure de paysage, 2000.

Bouillet, A., « Des sens à la sensibilité, quelle éducation ? ", Cahiers pédagogiques, n 374, Paris, 1999.

Brédif, H., «La qualité comme moyen de repenser le développement durable d'un territoire », EspacesTemps.net, Travaux, mai 2008, URL : https://www.espacestemps.net/articles/qualitedeveloppement-durable/.

Brédif, H., «Le vivant, les hommes et le territoire - Essai de biogéopolitique », thèse de doctorat, Institut national agronomique/École normale supérieure, 2004, URL : https:// www.researchgate.net/publication/ 29973265_Le_vivant_les_hommes_et_le_territoire_Essai_de_biogeopolitique. 
Calon, M., "Concevoir : modèle hiérarchique et modèle négocié ", dans Bonnet, M. (dir.), Actes du séminaire européen sur l'élaboration des projets architecturaux et urbains en Europe, Paris, Plan construction, 1997.

Claval, P., « Les géographes, le paysage et la modernisation », colloque de l'Union géographique internationale, Séoul, bulletin 50(2), 2000.

Claval, P., Géographie culturelle. Une approche des sociétés et des milieux, Paris, Armand Collin, coll. «U», 2012, $352 \mathrm{p}$.

Clette, V., Daems, A., Vandevyvere, A., « La ville au fil des pas : les marches d'exploration urbaine », Politique des Grandes Villes, Bruxelles, 2007, 41 p., URL : http://www.pyblik.brussels/ $\mathrm{nl} /$ node/2631.

Collectif IEP, « Diagnostic partagé du territoire sur le terrain », « Cadre de vie, répertoire d'outils ", Institut d'Éco-Pédagogie, 2011, URL : www.institut-eco-pedagogie.be/spip/spip.php ? article 341.

Comité directeur du Conseil de l'Europe sur la démocratie locale et régionale, « La participation des citoyens à la vie publique locale (rapport) ", $\mathrm{n}^{\circ} 72$, Conseil de l'Europe, coll. « Communes et régions d'Europe », 2000, p. 16 et sqq.

Conseil de l'Europe, Convention européenne du paysage, Florence, 2000.

Cordier, A., Géronnez, L., « Une reconnaissance mutuelle des capacités d'expertise de chacun - Le "Diagnostic Marchant" de "Paroles d'habitants", Bruxelles, Réseau Capacitation citoyenne, 2005, 64 p., URL : http://www.capacitation-citoyenne.org/livrets/sienne/diagMarch.pdf.

Cottereau, D., « Écoformation : entre soi et le monde », POUR, n 87, 2005, p. 111-117.

Dalimier, I., « Visite de terrain », dans « Tableau de bord "Participation et espaces publics" - Pour un développement et une gestion concertée des espaces publics ", rapport de recherche Topozym, Belspo, 2009, URL : http://www.topozym.ulg.ac.be/i/01-Visite\%20de\%20terrain.pdf.

Damien R., « Paysage et Citoyenneté ", dans Pons, G. (dir.), Le paysage : sauvegarde et création, Seyssel, Champ Vallon, 1999.

Dardel, E., L'Homme et la terre, Paris, Colin, 1952.

Declève, B., Forray, R., Michialino, P. (dir.), Coproduire nos espaces publics, Louvain-la-Neuve, UCL, Presses universitaires de Louvain, 2002, 195 p.

Gardner, H., Frames of Mind. The Theory of Multiple Intelligences (1983), New York, BasicBooks, 2003.

Guihéneuf, P.-Y., Cauchoix, F., Barret, P., Cayre, P. (coord.), La Formation au dialogue territorial. quelques clés issues d'une réflexion collective, Dijon, Educagri, 2006, 194 p.

Hansotte, M., Les Intelligences Citoyennes, Bruxelles, De Boeck Université, 2005.

Harou, R. (coord.), Fontaine, P., Rosinski, Z., « La participation des citoyens à la vie communale : enjeux et pratiques ", plaquette $n^{\circ} 3$, ministère de la Région wallonne, Namur, 2003, URL : https://cpdt.wallonie.be/sites/default/files/pdf/plaquette3.pdf.

Lardon, S., « Tisser des liens entre acteurs du territoire », dans Guihéneuf, P. Y., Cauchoix, F., Barret, P. et Cayre, R. (coord.), La Formation au dialogue territorial, Dijon, Educagri, 2006.

Lardon, S., Piveteau, V., « Méthodologies de diagnostic pour le projet de territoire : une approche par les modèles spatiaux », Geocarrefour, vol. 80/2, 2005, p. 75-90.

Lassus, B., « Le paysage comme organisation d'un référent sensible », Paris, Le Débat, n 65, 1991. 
Lazzarotti, O., « Le paysage, une fixation? », Cahiers de géographie du Québec, vol. 46, n 129, Université de Laval, 2002, p. 299-322.

Lipietz, A., « Le paysage, pays et visage : un point de vue écologiste », Monuments historiques, $\mathrm{n}^{\circ} 192,1994$.

Luginbühl, Y., « Symbolique et matérialité du paysage », Revue de l'économie méridionale, université de Montpellier III, vol. 46, nº 183, 1998, p. 235-245.

Loiseau, J.-M., Terrasson, F. et Trochel, Y., Le Paysage urbain, Paris, Sang de la Terre, 1993.

Michelin, Y. et Joliveau, T., « Le paysage au service de démarches participatives et prospectives de développement local : enseignements d'expériences de recherche-action conduites dans le Massif central ", Revue d'Auvergne, n 571, mai 2005, p. 233-262.

Moine, A., « Le territoire comme un système complexe : un concept opératoire pour l'aménagement et la géographie, L'Espace géographique, 2006/2, p. 115-132.

Ollagnon, H., « Une nécessaire rencontre des approches théoriques et pragmatiques de la gestion de la nature : l'audit patrimonial de type système-acteurs ", Cahier du Germes, nº 12, 1987.

Olmedo, E., « L'expérimentretien comme méthode d'enquête. Cartographie sensible et terrains de recherche collaboratifs entre art et géographie, M@ppemonde, nº 121, avril 2017, URL : http:// mappemonde.mgm.fr/121_img4/.

Orellana, I., «L'émergence de la communauté d'apprentissage ou l'acte de recréer des relations dialogiques et dialectiques de transformation du rapport au milieu de vie », dans Sauvé, L., Orellana, I., Van Steenberghe, E., « Éducation et environnement. Un croisement de savoirs », Cahiers scientifiques de l'ACFAS, $\mathrm{n}^{\circ}$ 104, 2005, p. 67-84.

Partoune, C., « Un modèle pédagogique global pour une approche du paysage fondée sur les nouvelles technologies de l'information et de la communication (NTIC) ", thèse de doctorat, 2004, URL : http://hdl.handle.net/2268/106995.

Partoune, C., « Développer une intelligence commune du territoire ", Éducation relative à l'environnement, regards, recherches, réflexions, vol. X, 2011-2012, p. 103-121.

Partoune, C., « Approche systémique et complexe », « Tableau de bord "Participation et espaces publics“ - Pour un développement et une gestion concertée des espaces publics », rapport de recherche Topozym, Belspo, 2009a., URL : http://www.topozym.ulg.ac.be/i/02Approche\%20syst\%C3\%A9mique\%20et\%20complexe.pdf.

Partoune, C., «Intelligence commune du territoire », 2009b, ibid., URL : http:// www.topozym.ulg.ac.be/i/16-Intelligence\%20commune\%20du\%20territoire.pdf.

Partoune, C., « Photolangage », 2009c, ibid., URL : http://www.topozym.ulg.ac.be/i/02-

Photolangage.pdf.

Partoune, C. et Ericx, M., « Hyperpaysage », 2009d, ibid., URL : http://www.topozym.ulg.ac.be/i/ 17-Hyperpaysage.pdf.

Partoune, C. et Ericx, M., « Les hyperpaysages : comment percevoir, interpréter et exprimer la complexité des sociétés derrière les paysages? », Bulletin de la Société géographique de l'université de Liège, 2005, n 45, p. 33-43.

Raffestin, C., « Théories du réel et géographicité », Espaces Temps, n 40-41, 1989, p. 26-31, URL : https://www.persee.fr/doc/espat_0339-3267_1989_num_40_1_3454.

Rosnay, J. de, Le Macroscope. Vers une vision globale, Paris, Seuil, coll. « Point », $\mathrm{n}^{\circ}$ 80, 1975, 346 p. 
Sauvé, L., « L'éducation relative à l'environnement : possibilités et contraintes », Connexion, vol. XXVII, $n^{\circ} 1 / 2,2002$, p. 1-4.

Sauvé, L. et Machabée, L., «La représentation : point focal de l'apprentissage ", Éducation relative à l'environnement - Regards, recherches, réflexions, vol. 2, 2000, p. 175-185.

Sauvé, L., Pour une éducation relative à l'environnement (1994), Montréal, éditions Guérin, coll. « Le défi éducatif », 1997, 361 p.

Scheers, L., «Éthique de l'intervention », « Tableau de bord "Participation et espaces publics“ Pour un développement et une gestion concertée des espaces publics », rapport de recherche Topozym, Belspo, 2009a, URL : http://www.topozym.ulg.ac.be/i/09-

Ethique\%20de\%20l'intervention.pdf.

Scheers L., « La motivation », 2009b, ibid., URL : http://www.topozym.ulg.ac.be/i/12La\%20motivation.pdf.

Sgard A., « Une « éthique du paysage » est-elle souhaitable? ", Vertigo. La revue électronique en sciences de l'environnement, vol. 10, $\mathrm{n}^{\circ} 1$, avril 2010, URL : http://journals.openedition.org/vertigo/ 9472.

Unesco-PNUE, Conférence intergouvernementale sur l'éducation relative à l'environnement, Tbilissi, 14 au 26 octobre 1977, rapport final, Paris.

Villemagne, $C .$, « Regard historique sur le développement de l'éducation relative à l'environnement ", dans La Branche, S. et Milot, N. (dir.), Enseigner les sciences sociales de l'environnement. Un manuel multidisciplinaire, Villeneuve-d'Ascq, Presses universitaires du Septentrion, 2010, p. 17-30.

\section{NOTES}

1. PNUD, Rapport mondial sur le développement humain 2003. Les objectifs du millénaire pour le développement: un pacte entre les pays pour vaincre la pauvreté humaine, Paris, Economica, $376 \mathrm{p}$.

2. PNUE, «L'Avenir de l'environnement mondial 3 », Londres, mars 2003.

3. Commission des Communautés européennes, "Gouvernance européenne, un livre blanc ", Bruxelles 2001.

4. www.educationpermanente.cfwb.be.

5. Décret du 21112013 relatif aux centres culturels, publié au Moniteur belge le 29012014 .

6. www.hyperpaysages.be.

7. www.topozym.ulg.ac.be.

8. www.topozym.ulg.ac.be/tableaubord.html.

9. Traduction par Christine Partoune de « an intelligence is the ability to solve problems, or to create products, that are valued within one or more cultural settings ».

10. https://enrd.ec.europa.eu/leader-clld_fr. 


\section{RÉSUMÉS}

L'article propose une analyse réflexive sur un dispositif de formation dédié à l'animation d'un diagnostic partagé du territoire au départ du paysage, proposé par l'Institut d'Éco-Pédagogie, association située à Liège sans but lucratif spécialisée en éducation relative à l'environnement reconnue en éducation permanente. Le cadre de référence dont les formateurs s'inspirent repose sur les fondements de l'éducation relative à l'environnement et sur les enjeux d'une éducation relative au paysage, ainsi que sur le concept d'intelligence commune du territoire. Le modèle de formation qui se dégage de l'analyse du dispositif est constitué de trois étapes clés : une phase d'immersion visant la mise en relation des personnes avec elles-mêmes, avec les autres et avec leur milieu de vie; une phase d'éveil et de prise de conscience de soi au monde; une phase de diagnostic du territoire intégrant une approche systémique et complexe du milieu de vie, ainsi que l'émancipation socioculturelle des citoyens. Les points forts et les limites de ces dispositifs sont ensuite discutés.

The article offers a reflexive analysis of a training process dedicated to a shared diagnosis of a territory based on the landscape, proposed by the Institut d'Eco-Pédagogie, a non-profit association situated in Liège specialised in environmental education and acknowledged in the field of continuous training. The frame of reference for the teachers is environmental education and education to the landscape, as well as the concept of a common intelligence of a territory. The training model resulting from this process comprises three key stages : an immersion phase for participants to relate to themselves, other participants and their living environment ; a phase for developing awareness of self in relation to the world; and a phase for making an appraisal of the territory integrating a complex systemic approach of the living environment and a socio-cultural empowerment of the citizen. The strong and weak points of these methods are then discussed.

\section{INDEX}

Mots-clés : paysage, intelligence commune du territoire, éducation relative à l'environnement, sensibilisation, diagnostic territorial

Keywords : landscape, common intelligence of a territory, environmental education, awarenessbuilding, territorial evaluation

\section{AUTEUR}

\section{CHRISTINE PARTOUNE}

Chargée de cours en didactique de la géographie et directrice du Laboratoire de méthodologie de la géographie de l'université de Liège (Belgique), Christine Partoune est membre de l'Unité de recherche en didactique et formation des enseignants (DIDACTIfen) et présidente de l'Institut d'Éco-Pédagogie (www.institut-eco-pedagogie.be), association sans but lucratif située également à Liège.

c.partoune[at]uliege[dot]be www.lmg.ulg.ac.be 